\title{
TWO LIMIT TRANSITIONS INVOLVING MULTIVARIABLE BC TYPE ASKEY-WILSON POLYNOMIALS
}

\author{
JASPER V. STOKMAN \\ Department of Mathematics, University of Amsterdam \\ Plantage Muidergracht 24, 1018 TV Amsterdam, The Netherlands \\ E-mail: jasper@fwi.uva.nl
}

\begin{abstract}
In the first part (without proofs) an orthogonality measure with partly discrete and partly continuous support will be introduced for the five parameter family of multivariable $\mathrm{BC}$ type Askey-Wilson polynomials. In the second part, the limit transitions from $\mathrm{BC}$ type Askey-Wilson polynomials to BC type big and little q-Jacobi polynomials will be described in detail.
\end{abstract}

1. Introduction. Recently, part of the Askey-Wilson scheme for one variable basic hypergeometric orthogonal polynomials has been generalized to a multivariable BC type Askey-Wilson scheme. The starting point was the introduction of families of orthogonal polynomials for general root systems by Macdonald in $[\mathrm{M}]$. For BC, this yielded a three parameter family of orthogonal polynomials, generalizing part of the four parameter family of one variable Askey-Wilson polynomials. Koornwinder in [K1] extended this family to a five parameter family of multivariable BC type orthogonal polynomials. Four parameters $a, b, c, d$ play the same role as in the one variable case, and the fifth parameter $t$ is an extra deformation parameter. Furthermore, multivariable BC type big and little q-Jacobi polynomials were introduced in [S], and limit transitions between these three families have been studied in [SK].

In the one variable case, the limit transition from Askey-Wilson polynomials to big resp. little q-Jacobi polynomials has a very special feature: the Askey-Wilson polynomials which are involved in the limit transition are orthogonal with respect to a measure with support consisting of a continuous and a discrete part. In the limit, the continuous part of the orthogonality measure shrinks to $\{0\}$ (with weight tending to zero), while the discrete

1991 Mathematics Subject Classification: 33D45, 33D80.

Key words and phrases: BC type Askey-Wilson polynomials, BC type big and little q-Jacobi polynomials, multivariable orthogonal polynomials, second order q-difference operators.

The paper is in final form and no version of it will be published elsewhere. 
part tends to the infinite set of discrete mass points corresponding to the big resp. little q-Jacobi polynomials (cf. [K2]).

The orthogonality measure for the multivariable BC type Askey-Wilson polynomials has been introduced in [K1] for parameters $a, b, c, d$ such that the measure consists only of a continuous part. Consequently, the limit transition from the multivariable AskeyWilson polynomials to the multivariable big resp. little q-Jacobi polynomials which was proved in $[\mathrm{SK}]$, uses a definition of the multivariable Askey-Wilson polynomials for more general parameter values by rational extension in the parameters, without knowing the orthogonality measure for the general parameter values. In particular, an interpretation of the limit transitions in terms of the supports of the orthogonality measures could not be given in the multivariable case.

In the second section, the orthogonality measure for the multivariable BC type AskeyWilson polynomials will be given for more general parameter values. This gives rise to extra discrete parts in the orthogonality measure. Proofs are omitted at this stage, they will be given in [S1].

Knowing the orthogonality measure for the larger parameter domain allows us to give an interpretation of the limit transition from multivariable Askey-Wilson polynomials to multivariable big resp. little q-Jacobi polynomials in terms of the supports of the orthogonality measures. This will be discussed in detail in section 3 .

We will restrict to the case that the deformation parameter $t$ is $q^{k}$ for $k$ a natural number.

Notations: $\mathbf{N}=\{1,2, \ldots\}$ will be the natural numbers and $\mathbf{N}_{0}$ the natural numbers together with 0. Empty sums are equal to 0, empty products are equal to 1.

Acknowledgement: The author thanks Prof. T.H. Koornwinder for useful comments on an earlier version of the paper.

2. The orthogonality measure for BC type Askey-Wilson polynomials. In this section we fix $q \in(0,1)$.

We first introduce some notations. The q-shifted factorial is given by

$$
(a ; q)_{i}:=\prod_{j=0}^{i-1}\left(1-q^{j} a\right) \quad\left(i \in \mathbf{N}_{0}\right), \quad(a ; q)_{\infty}:=\prod_{j=0}^{\infty}\left(1-q^{j} a\right),
$$

and we denote

$$
\left(a_{1}, \ldots, a_{m} ; q\right)_{i}:=\prod_{j=0}^{m}\left(a_{j} ; q\right)_{i} \quad\left(i \in \mathbf{N}_{0}\right), \quad\left(a_{1}, \ldots, a_{m} ; q\right)_{\infty}:=\prod_{j=0}^{m}\left(a_{j} ; q\right)_{\infty} .
$$

We write

$$
{ }_{r+1} \phi_{r}\left[\begin{array}{c}
a_{1}, \ldots, a_{r+1} \\
b_{1}, \ldots, b_{r}
\end{array} ; q, x\right]:=\sum_{k=0}^{\infty} \frac{\left(a_{1}, \ldots, a_{r+1} ; q\right)_{k} x^{k}}{\left(q, b_{1}, \ldots, b_{r} ; q\right)_{k}}
$$

for the q-hypergeometric series. Let $N \in \mathbf{Z}$. Define for a function $f: \mathbf{C} \rightarrow \mathbf{C}$ and $\alpha, \beta \in \mathbf{C}$ the Jackson q-integral of $f$, truncated at $N$, by

$$
\int_{\alpha}^{\beta} f(x) d_{q, N} x:=\int_{0}^{\beta} f(x) d_{q, N} x-\int_{0}^{\alpha} f(x) d_{q, N} x
$$




$$
\begin{gathered}
\int_{0}^{\beta} f(x) d_{q, N} x:=\sum_{k=0}^{N} f\left(\beta q^{k}\right)\left(\beta q^{k}-\beta q^{k+1}\right) \text { if } N \geq 0 \\
\int_{\alpha}^{\beta} f(x) d_{q, N} x:=0 \text { if } N<0 .
\end{gathered}
$$

The Jackson q-integral for (continuous) functions $f$ is defined by

$$
\int_{\alpha}^{\beta} f(x) d_{q} x:=\lim _{N \rightarrow \infty} \int_{\alpha}^{\beta} f(x) d_{q, N} x .
$$

Let $S_{n}$ be the symmetric group (group of permutations of the set $\{1, \ldots, n\}$ ). Let $\mathcal{W}$ be the semidirect product of $\{ \pm 1\}^{n}$ and $S_{n}$. Let $z_{1}, \ldots, z_{n}$ be independent variables, then $\mathcal{W}$ acts on the algebra $A:=\mathbf{C}\left[z_{1}^{ \pm 1}, \ldots, z_{n}^{ \pm 1}\right]$ by inversions and permutations of the variables. Denote $A^{W}$ for the subalgebra of $\mathcal{W}$-invariant functions in $A$. A basis for $A^{W}$ is given by the monomials $\left\{m_{\lambda} / \lambda \in P^{+}\right\}$, where $P^{+}:=\left\{\mu \in \mathbf{N}_{0}^{n} / \mu_{1} \geq \ldots \geq \mu_{n}\right\}$, and

$$
m_{\lambda}(z):=\sum_{\mu \in \mathcal{W} \lambda} z^{\mu}
$$

with $z^{\mu}=z_{1}^{\mu_{1}} \ldots z_{n}^{\mu_{n}}$. The $\mathcal{W}$-orbit of $\lambda \in P^{+} \subset \mathbf{Z}^{n}$ is with respect to the natural action of $\mathcal{W}$ on $\mathbf{Z}^{n}$.

In this section, we will define a scalar product on $A^{W}$ depending on parameters $a, b, c, d, t$ satisfying $t=q^{k}, k \in \mathbf{N}$, and $(a, b, c, d) \in V_{A W}$ with $V_{A W}$ given by

Definition 2.1 (Parameter domain for the Askey-Wilson polynomials). Let $V_{A W}$ be the set of parameters $(a, b, c, d)$ which satisfy the following conditions:

(1) $a, b, c, d$ are real, or if complex, then they appear in conjugate pairs.

(2) $a b, a c, a d, b c, b d, c d \notin \mathbf{R}_{\geq 1}:=\{r \in \mathbf{R} / r \geq 1\}$.

Note that if $(a, b, c, d) \in V_{A W}$ and $e \in\{a, b, c, d\}$ with $|e| \geq 1$, then $e \in \mathbf{R}$. Furthermore, at most two of the four parameters $a, b, c, d$ have modulus $\geq 1$; If there are two, then one is $\geq 1$, and the other is $\leq-1$.

Let $(a, b, c, d) \in V_{A W}$. For $e \in\{a, b, c, d\}$ with $|e|>1$, let $N_{e} \in \mathbf{Z}$ be the largest integer such that $\left|e q^{N_{e}}\right|>1$. Take $N_{e}:=-1$ for $e \in\{a, b, c, d\}$ with $|e| \leq 1$. Let $T_{k}$ be the $k$-torus, given as the $k$-fold direct product of the unit circle $\mathcal{C}$ in $\mathbf{C}^{k}$ :

$$
T_{k}:=\left\{\left(w_{1}, \ldots, w_{k}\right) \in \mathbf{C}^{k} /\left|w_{i}\right|=1(i=1, \ldots, k)\right\} .
$$

Give $T_{k}$ the orientation induced from the counterclockwise orientation on each unit circle $\left\{w_{i} /\left|w_{i}\right|=1\right\}$. Define for $m \in\{0, \ldots, n\}$ a hermitian form $\langle., .\rangle_{m, q, t}^{a, b, c, d}: A^{W} \times A^{W} \rightarrow \mathbf{C}$ by:

$$
\begin{aligned}
\left\langle p_{1}, p_{2}\right\rangle_{m}:= & \frac{2^{m}\left(\begin{array}{c}
n \\
m
\end{array}\right)}{(2 \pi i)^{n-m}} \sum_{e_{1}, \ldots, e_{m}} \int_{z_{1}=0}^{e_{1}} \ldots \int_{z_{m}=0}^{e_{m}} \int . \cdot \int_{\left(z_{m+1}, \ldots, z_{n}\right) \in T_{n-m}} \\
& p_{1}(z) \overline{p_{2}(z)} \Delta_{A W, m}(z) \frac{d_{q, N_{e_{1}}} z_{1}}{(1-q) z_{1}} \ldots \frac{d_{q, N_{e_{m}}} z_{m}}{(1-q) z_{m}} \frac{d z_{m+1}}{z_{m+1}} \ldots \frac{d z_{n}}{z_{n}}
\end{aligned}
$$

for $p_{1}, p_{2} \in A^{W}$. The sum is taken over $e_{j} \in\{a, b, c, d\}$ for all $j=1, \ldots, m$, so a sum with at least one $\left|e_{j}\right| \leq 1$ gives zero contribution. For $m=0$ we can skip the summation 
sign and integrate over $\left(z_{1}, \ldots, z_{n}\right) \in T_{n}$. The weight function $\Delta_{A W, m}\left(z ; a, b, c, d ; q, q^{k}\right)$ is defined by

$$
\Delta_{A W, m}(z):=\left(\prod_{l=1}^{m} w_{A W, d}\left(z_{l}\right)\right)\left(\prod_{r=m+1}^{n} w_{A W, c}\left(z_{r}\right)\right) \delta_{k}(z)
$$

with

$$
\delta_{k}(z):=\prod_{1 \leq i<j \leq n}\left(z_{i} z_{j}, z_{i} z_{j}^{-1}, z_{i}^{-1} z_{j}, z_{i}^{-1} z_{j}^{-1} ; q\right)_{k},
$$

and $w_{A W, c}(x ; a, b, c, d ; q)$, resp. $w_{A W, d}(x ; a, b, c, d ; q)$ given by

$$
w_{A W, c}(x ; a, b, c, d ; q):=\frac{\left(x^{2}, x^{-2} ; q\right)_{\infty}}{\left(a x, a x^{-1}, b x, b x^{-1}, c x, c x^{-1}, d x, d x^{-1} ; q\right)_{\infty}}
$$

and for $e \in\{a, b, c, d\}$ and $i \in\left\{0, \ldots, N_{e}\right\}$,

$$
\begin{aligned}
w_{A W, d}\left(e q^{i} ; e ; f, g, h ; q\right):= & \frac{\left(e^{-2} ; q\right)_{\infty}}{(q, e f, f / e, e g, g / e, e h, h / e ; q)_{\infty}} \times \\
& \frac{\left(e^{2}, e f, e g, e h ; q\right)_{i}}{(q, e q / f, e q / g, e q / h ; q)_{i}} \frac{\left(1-e^{2} q^{2 i}\right)}{\left(1-e^{2}\right)}\left(\frac{q}{e f g h}\right)^{i}
\end{aligned}
$$

with $f, g, h \in \mathbf{C}$ such that $e, f, g, h$ is a permutation of $a, b, c, d$. Then the hermitian form $\langle., .\rangle_{A W, q, t}^{a, b, c, d}$ is defined by

$$
\left\langle p_{1}, p_{2}\right\rangle_{A W}:=\sum_{m=0}^{n}\left\langle p_{1}, p_{2}\right\rangle_{m} \quad\left(p_{1}, p_{2} \in A^{W}\right) .
$$

Note that the measure $\frac{d_{q, N_{e}} x}{(1-q) x}$ on $[0, e]$ with $|e|>1$ is just the counting measure for the set $\left\{e, \ldots, e q^{N_{e}}\right\}$, i.e.

$$
\int_{x=0}^{e} f(x) \frac{d_{q, N_{e}} x}{(1-q) x}=\sum_{k=0}^{N_{e}} f\left(e q^{k}\right) .
$$

Denote $W_{A W, m}\left(a, b, c, d ; q, q^{k}\right)$ for the integration domain of $\langle., .\rangle_{m}$. So we have $W_{A W, 0}=$ $T_{n}$, and for $m>0$ we have

$$
\begin{array}{r}
W_{A W, m}=\left\{\left(z_{1}, \ldots, z_{n}\right) \in \mathbf{C}^{n} / z_{i} \in \bigcup_{e:|e|>1}\left\{e, \ldots, e q^{N_{e}}\right\} \quad(i=1, \ldots, m)\right. \\
\text { and } \left.\left(z_{m+1}, \ldots, z_{n}\right) \in T_{n-m}\right\}
\end{array}
$$

if there exists an $e \in\{a, b, c, d\}$ with $|e|>1$, and $W_{A W, m}=\emptyset$ otherwise.

The hermitian form $\langle., .\rangle_{A W}$ is positive definite due to following lemma.

Lemma 2.2. Let $q \in(0,1),(a, b, c, d) \in V_{A W}$ and $t=q^{k}$ with $k \in \mathbf{N}$.

(a) $\Delta_{A W, m}(z) \geq 0$ for $z \in W_{A W, m}$ and $m \in\{0, \ldots, n\}$.

(b) Let $0<m<n$. Let $z_{i} \in \cup_{e:|e|>1}\left\{e, \ldots, e q^{N_{e}}\right\}$ for $i=1 \ldots, m$.

Then $\Delta_{A W, m}\left(z_{1}, \ldots, z_{m}, w_{m+1}, \ldots, w_{n}\right)=0$ for all $\left(w_{m+1}, \ldots, w_{n}\right) \in T_{n-m}$ if and only if $z_{i}=q^{l} z_{j}$ for certain $l \in\{0, \ldots, k-1\}$ and certain $i, j \in\{1, \ldots, m\}, i \neq j$.

(c) Let $z \in W_{A W, n}$, then $\Delta_{A W, n}(z)=0$ if and only if $z_{i}=q^{l} z_{j}$ for certain $l \in\{0, \ldots, k-1\}$ and certain $i, j \in\{1, \ldots, n\}, i \neq j$. 
Define a partial order on $P^{+}$by

$$
\mu \leq \lambda \Leftrightarrow \sum_{l=1}^{j} \mu_{l} \leq \sum_{l=1}^{j} \lambda_{l} \quad \forall j=1, \ldots, n
$$

for $\lambda, \mu \in P^{+}$.

Definition 2.3. Let $(a, b, c, d) \in V_{A W}$ and $t=q^{k}, k \in \mathbf{N}$.

The Askey-Wilson polynomials $\left\{P_{\lambda}^{A W}(z ; a, b, c, d ; q, t) / \lambda \in P^{+}\right\}$are defined by the following two conditions:

(1) $P_{\lambda}^{A W}=m_{\lambda}+\sum_{\mu<\lambda} c_{\lambda, \mu} m_{\mu}$ for certain $c_{\lambda, \mu} \in \mathbf{C}$,

(2) $\left\langle P_{\lambda}^{A W}, m_{\mu}\right\rangle_{A W}=0$ for $\mu<\lambda$.

Conditions (1) and (2) uniquely determine $P_{\lambda}^{A W}$ and $\left\{P_{\lambda}^{A W} / \lambda \in P^{+}\right\}$is a basis of $A^{W}$. Full orthogonality of the Askey-Wilson polynomials with respect to $\langle., .\rangle_{A W}$ is not obvious since the order $(\leq)$ on $P^{+}$is a partial order. It is clear that $\left\langle P_{\lambda}^{A W}, P_{\mu}^{A W}\right\rangle_{A W}=0$ if $\mu<\lambda$ or if $\mu>\lambda$.

In the one variable case, the Askey-Wilson polynomials defined by definition 2.3 are independent of $t$ and correspond with the monic one variable Askey-Wilson polynomials

$$
P_{l}^{A W}(x ; a, b, c, d ; q):=\frac{(a b, a c, a d ; q)_{l}}{a^{l}\left(q^{l-1} a b c d ; q\right)_{l}}{ }_{4} \phi_{3}\left[\begin{array}{c}
q^{-l}, q^{l-1} a b c d, a x, a x^{-1} \\
a b, a c, a d
\end{array} ; q, q\right] \quad\left(l \in \mathbf{N}_{0}\right)
$$

(cf. [AW], theorem 2.4. The term $\frac{\left(1-a q^{2 k}\right)}{(1-a)}$ in $[\mathrm{AW}]$ formula (2.10) should be replaced by $\frac{\left(1-a^{2} q^{2 k}\right)}{\left(1-a^{2}\right)}$, so the discrete weights $w_{A W, d}$ coincide with the weights given by $(2.10)$ in $[\mathrm{AW}])$. Note that $P_{l}^{A W}\left(l \in \mathbf{N}_{0}\right)$ is a monic polynomial of degree $l$ in the variable $x+x^{-1}$. Usely, the one variable Askey-Wilson polynomials are normalized differently (see $[\mathrm{AW}]$ ).

Next we introduce a second order q-difference operator for which the Askey-Wilson polynomials are joint eigenfunctions. The second order q-difference operator $D_{A W, q, t}^{a, b, c, d}$ is defined by (cf. [K1]):

$$
D_{A W}:=\sum_{j=1}^{n}\left(\phi_{A W, j}^{+}(z)\left(T_{q, j}-\mathrm{Id}\right)+\phi_{A W, j}^{-}(z)\left(T_{q^{-1}, j}-\mathrm{Id}\right)\right),
$$

with $T_{q^{ \pm 1}, j}$ the $q^{ \pm 1}$-shift in the $j^{\text {th }}$-coordinate:

$$
\left(T_{q^{ \pm 1}, j} f\right)(z):=f\left(z_{1}, \ldots, z_{j-1}, q^{ \pm 1} z_{j}, z_{j+1}, \ldots, z_{n}\right)
$$

and $\phi_{A W, j}^{+}(z ; a, b, c, d ; q, t)$ and $\phi_{A W, j}^{-}(z ; a, b, c, d ; q, t)$ are given by

$$
\begin{gathered}
\phi_{A W, j}^{+}(z):=\frac{\left(1-a z_{j}\right)\left(1-b z_{j}\right)\left(1-c z_{j}\right)\left(1-d z_{j}\right)}{\left(1-z_{j}^{2}\right)\left(1-q z_{j}^{2}\right)} \prod_{l \neq j} \frac{\left(1-t z_{l} z_{j}\right)\left(1-t z_{l}^{-1} z_{j}\right)}{\left(1-z_{l} z_{j}\right)\left(1-z_{l}^{-1} z_{j}\right)} \\
\phi_{A W, j}^{-}(z):=\frac{\left(a-z_{j}\right)\left(b-z_{j}\right)\left(c-z_{j}\right)\left(d-z_{j}\right)}{\left(1-z_{j}^{2}\right)\left(q-z_{j}^{2}\right)} \prod_{l \neq j} \frac{\left(t-z_{l} z_{j}\right)\left(t-z_{l}^{-1} z_{j}\right)}{\left(1-z_{l} z_{j}\right)\left(1-z_{l}^{-1} z_{j}\right)} .
\end{gathered}
$$

Define for $\lambda \in P^{+}$,

$$
E_{\lambda}^{A W}(a, b, c, d ; q, t):=\sum_{j=1}^{n}\left(q^{-1} a b c d t^{2 n-j-1}\left(q^{\lambda_{j}}-1\right)+t^{j-1}\left(q^{-\lambda_{j}}-1\right)\right) .
$$


We have the following theorem.

TheOREM 2.4. Let $q \in(0,1),(a, b, c, d) \in V_{A W}$ and $t=q^{k}$, with $k \in \mathbf{N}$.

(a) For all $\lambda \in P^{+}$we have

$$
D_{A W} P_{\lambda}^{A W}=E_{\lambda}^{A W} P_{\lambda}^{A W}
$$

(b) Let $\lambda, \mu \in P^{+}$with $\lambda \neq \mu$. We have

$$
\left\langle P_{\lambda}^{A W}, P_{\mu}^{A W}\right\rangle_{A W}=0 .
$$

If $(a, b, c, d) \in V_{A W}$ with $|e| \leq 1$ for all $e \in\{a, b, c, d\}$ then the Askey-Wilson polynomials given by definition 2.3 are exactly the multivariable Askey-Wilson polynomials with $t=q^{k}\left(k \in \mathbf{N}_{0}\right)$ defined by Koornwinder in [K1], since the orthogonality measure in this case (scalar product given by integration over the torus $T_{n}$ with weight function $\left.\Delta_{A W, 0}(z)\right)$ coincides with the orthogonality measure defined in [K1]. Koornwinder proved theorem 2.4 for $(a, b, c, d) \in V_{A W}$ with $|e| \leq 1$ for all $e \in\{a, b, c, d\}$ with continuous parameter $t \in(-1,1)$, for suitable scalar product $\langle., .\rangle_{A W, t}$ (cf. [K1]).

3. Limit transitions to BC type big and little q-Jacobi polynomials. We first recapitulate the definition of multivariable big and little q-Jacobi polynomials for the special case that $t=q^{k}, k \in \mathbf{N}$ (cf. [S]). Let $c, d>0$, and

$$
a \in\left(\frac{-c}{d q}, \frac{1}{q}\right), b \in\left(\frac{-d}{c q}, \frac{1}{q}\right),
$$

or $a=c z, b=-d \bar{z}$ with $z \in \mathbf{C} \backslash \mathbf{R}$. Denote $V_{B}^{q}$ for the set of parameters $(a, b, c, d)$ which satisfy these conditions. Let $A^{S}:=\mathbf{C}\left[z_{1}, \ldots, z_{n}\right]^{S_{n}}$ be the algebra of symmetric polynomials, and $\left\{\tilde{m}_{\lambda}(z) / \lambda \in P^{+}\right\}$the basis of monomials for $A^{S}$ :

$$
\tilde{m}_{\lambda}(z):=\sum_{\mu \in S_{n} \lambda} z^{\mu}
$$

Define for $q \in(0,1),(a, b, c, d) \in V_{B}^{q}$ and $t=q^{k}$ with $k \in \mathbf{N}$ a hermitian form $\langle., .\rangle_{B, q, t}^{a, b, c, d}$ on $A^{S}$ by

$$
\langle f, g\rangle_{B}=\int_{z_{1}=-d}^{c} \int_{z_{2}=-d}^{c} \ldots \int_{z_{n}=-d}^{c} f(z) \overline{g(z)} \Delta_{B}(z) d_{q} z_{1} \ldots d_{q} z_{n}
$$

for $f, g \in A^{S}$ with weight function $\Delta_{B}\left(z ; a, b, c, d ; q, q^{k}\right)$ given by

$$
\Delta_{B}(z)=\left(\prod_{i=1}^{n} w_{B}\left(z_{i}\right)\right) \Delta(z) \prod_{1 \leq i<j \leq n} z_{i}^{2 k-1}\left(q^{1-k} \frac{z_{j}}{z_{i}} ; q\right)_{2 k-1},
$$

with $\Delta(z):=\prod_{1 \leq i<j \leq n}\left(z_{i}-z_{j}\right)$ the Vandermonde determinant and $w_{B}(x ; a, b, c, d ; q)$ the weight function involved in the orthogonality measure for the one variable big q-Jacobi polynomials:

$$
w_{B}(x ; a, b, c, d ; q):=\frac{(q x / c,-q x / d ; q)_{\infty}}{(q a x / c,-q b x / d ; q)_{\infty}} .
$$

The integration domain $W_{B}\left(a, b, c, d ; q, q^{k}\right)$ of $\langle., .\rangle_{B}$ is given by

$$
W_{B}:=\left\{z \in \mathbf{R}^{n} / z_{i} \in\left\{-d q^{k}\right\}_{k \in \mathbf{N}_{0}} \cup\left\{c q^{l}\right\}_{l \in \mathbf{N}_{0}} \quad(i=1, \ldots, n)\right\} .
$$


We have

Lemma 3.1. Let $q \in(0,1),(a, b, c, d) \in V_{B}^{q}$ and $t=q^{k}, k \in \mathbf{N}$.

(a) $\Delta_{B}(z) \geq 0$ for all $z \in W_{B}$.

(b) $\Delta_{B}(z)=0$ for $z \in W_{B}$ if and only if $z_{i}=q^{l} z_{j}$ for certain $l \in\{0, \ldots, k-1\}$ and certain $i, j \in\{1, \ldots, n\}, i \neq j$.

In particular, $\langle., .\rangle_{B}$ is positive definite.

Definition 3.2. Let $q \in(0,1),(a, b, c, d) \in V_{B}^{q}$ and $t=q^{k}$ with $k \in \mathbf{N}$.

The big q-Jacobi polynomials $\left\{P_{\lambda}^{B}(. ; a, b, c, d ; q, t) / \lambda \in P^{+}\right\}$are defined by the following two conditions:

(1) $P_{\lambda}^{B}=\tilde{m}_{\lambda}+\sum_{\mu<\lambda} c_{\lambda, \mu} \tilde{m}_{\mu}$ for certain $c_{\lambda, \mu} \in \mathbf{C}$,

(2) $\left\langle P_{\lambda}^{B}, \tilde{m}_{\mu}\right\rangle_{B}=0$ for $\mu<\lambda$.

For the little q-Jacobi polynomials, assume $a \in(0,1 / q)$ and $b \in(-\infty, 1 / q)$, and denote $V_{L}^{q}$ for the set of parameters $(a, b)$ which satisfy these conditions. Define for $q \in(0,1)$, $(a, b) \in V_{L}^{q}$ and $t=q^{k}$ with $k \in \mathbf{N}$ a hermitian form $\langle., .\rangle_{L, q, t}^{a, b}$ on $A^{S}$ by

$$
\langle f, g\rangle_{L}=\int_{z_{1}=0}^{1} \ldots \int_{z_{n}=0}^{1} f(z) \overline{g(z)} \Delta_{L}(z) d_{q} z_{1} \ldots d_{q} z_{n}
$$

for $f, g \in A^{S}$ with weight function $\Delta_{L}\left(z ; a, b ; q, q^{k}\right)$ :

$$
\Delta_{L}(z)=\left(\prod_{i=1}^{n} w_{L}\left(z_{i}\right)\right) \Delta(z) \prod_{1 \leq i<j \leq n} z_{i}^{2 k-1}\left(q^{1-k} \frac{z_{j}}{z_{i}} ; q\right)_{2 k-1},
$$

and $w_{L}(x ; a, b ; q)$ the weight function involved in the orthogonality measure for the one variable little q-Jacobi polynomials:

$$
w_{L}(x ; a, b ; q):=\frac{(q x ; q)_{\infty}}{(q b x ; q)_{\infty}} x^{\alpha} \quad\left(a=q^{\alpha}\right) .
$$

The integration domain $W_{L}\left(a, b ; q, q^{k}\right)$ of $\langle., .\rangle_{L}$ is given by

$$
W_{L}:=\left\{z \in \mathbf{R}^{n} / z_{i} \in\left\{q^{k}\right\}_{k \in \mathbf{N}_{0}} \quad(i=1, \ldots, n)\right\} .
$$

We have

Lemma 3.3. Let $q \in(0,1),(a, b) \in V_{L}^{q}$ and $t=q^{k}, k \in \mathbf{N}$.

(a) $\Delta_{L}(z) \geq 0$ for all $z \in W_{L}$.

(b) $\Delta_{L}(z)=0$ for $z \in W_{L}$ if and only if $z_{i}=q^{l} z_{j}$ for certain $l \in\{0, \ldots, k-1\}$ and certain $i, j \in\{1, \ldots, n\}, i \neq j$.

In particular, $\langle., .\rangle_{L}$ is positive definite.

Definition 3.4. Let $q \in(0,1),(a, b) \in V_{L}^{q}$ and $t=q^{k}, k \in \mathbf{N}$.

The little $q$-Jacobi polynomials $\left\{P_{\lambda}^{L}(. ; a, b ; q, t) / \lambda \in P^{+}\right\}$are defined by the following two conditions:

(1) $P_{\lambda}^{L}=\tilde{m}_{\lambda}+\sum_{\mu<\lambda} c_{\lambda, \mu} \tilde{m}_{\mu}$ for certain $c_{\lambda, \mu} \in \mathbf{C}$,

(2) $\left\langle P_{\lambda}^{L}, \tilde{m}_{\mu}\right\rangle_{L}=0$ for $\mu<\lambda$. 
The second order q-diference operator which has the big resp. little q-Jacobi polynomials as mutual eigenfunctions is given by

$$
\begin{aligned}
D_{B} & =\sum_{j=1}^{n}\left(\phi_{B, j}^{+}(z)\left(T_{q, j}-\mathrm{Id}\right)+\phi_{B, j}^{-}(z)\left(T_{q^{-1}, j}-\mathrm{Id}\right)\right), \\
D_{L} & =\sum_{j=1}^{n}\left(\phi_{L, j}^{+}(z)\left(T_{q, j}-\mathrm{Id}\right)+\phi_{L, j}^{-}(z)\left(T_{q^{-1}, j}-\mathrm{Id}\right)\right),
\end{aligned}
$$

with $\phi_{B, j}^{+}(z ; a, b, c, d ; q, t)$ given by

$$
\phi_{B, j}^{+}(z):=q t^{n-1}\left(a-\frac{c}{q z_{j}}\right)\left(b+\frac{d}{q z_{j}}\right) \prod_{l \neq j} \frac{z_{l}-t z_{j}}{z_{l}-z_{j}}
$$

and $\phi_{B, j}^{-}(z ; a, b, c, d ; q, t)$ given by

$$
\phi_{B, j}^{-}(z):=\left(1-\frac{c}{z_{j}}\right)\left(1+\frac{d}{z_{j}}\right) \prod_{l \neq j} \frac{z_{j}-t z_{l}}{z_{j}-z_{l}}
$$

respectively

$$
\begin{aligned}
& \phi_{L, j}^{+}(z ; a, b ; q, t):=\phi_{B, j}^{+}(z ; b, a, 1,0 ; q, t), \\
& \phi_{L, j}^{-}(z ; a, b ; q, t):=\phi_{B, j}^{-}(z ; b, a, 1,0 ; q, t) .
\end{aligned}
$$

Define for $\lambda \in P^{+}, E_{\lambda}^{B, L}(a, b ; q, t)$ by

$$
E_{\lambda}^{B, L}=\sum_{j=1}^{n}\left(q a b t^{2 n-j-1}\left(q^{\lambda_{j}}-1\right)+t^{j-1}\left(q^{-\lambda_{j}}-1\right)\right) .
$$

In $[S]$, the following theorem was proved:

TheOREM 3.5. Let $q \in(0,1)$.

(a) Let $(a, b, c, d) \in V_{B}^{q}$ and $t=q^{k}, k \in \mathbf{N}$. $P_{\lambda}^{B}$ is an eigenfunction of $D_{B}$ with eigenvalue $E_{\lambda}^{B, L}$ for all $\lambda \in P^{+}$and $\left\{P_{\mu}^{B} / \mu \in P^{+}\right\}$is an orthogonal basis with respect to $\langle., .\rangle_{B}$.

(b) Let $(a, b) \in V_{L}^{q}$ and $t=q^{k}, k \in \mathbf{N}$. $P_{\lambda}^{L}$ is an eigenfunction of $D_{L}$ with eigenvalue $E_{\lambda}^{B, L}$ for all $\lambda \in P^{+}$and $\left\{P_{\mu}^{L} / \mu \in P^{+}\right\}$is an orthogonal basis with respect to $\langle., .\rangle_{L}$.

In fact, this theorem was proved in [S] for arbitrary $t \in(0,1)$, for suitable scalar products $\langle., .\rangle_{B, t}$ and $\langle., .\rangle_{L, t}$.

Denote $|\lambda|:=\sum_{i=1}^{n} \lambda_{i}$ for $\lambda \in P^{+}$, and $c z:=\left(c z_{1}, \ldots, c z_{n}\right)$ for $z=\left(z_{1}, \ldots, z_{n}\right)$, $c \in$ C. Let $\phi_{\epsilon}, \phi$ be Laurent polynomials in the variables $z_{1}, \ldots, z_{n}$, then we say that

$$
\lim _{\epsilon \rightarrow 0} \phi_{\epsilon}=\phi
$$

if

$$
\lim _{\epsilon \rightarrow 0} \phi_{\epsilon}(z)=\phi(z) \quad \forall z \in(\mathbf{C} \backslash\{0\})^{n} .
$$

We have the following limit transitions from Askey-Wilson polynomials to the big resp. little q-Jacobi polynomials. 
Theorem 3.6. Fix $\lambda \in P^{+}$.

(a) Let $q \in(0,1)$ and $k \in \mathbf{N}$. Suppose that $(a, b, c, d) \in V_{B}^{q}$, then

$$
\begin{array}{r}
\lim _{\epsilon \downarrow 0}\left(\frac{\epsilon(c d)^{\frac{1}{2}}}{q^{\frac{1}{2}}}\right)^{|\lambda|} P_{\lambda}^{A W}\left(\frac{q^{\frac{1}{2}} z}{\epsilon(c d)^{\frac{1}{2}}} ;\right. \\
\quad \epsilon q^{\frac{1}{2}} a(d / c)^{\frac{1}{2}}, \epsilon^{-1} q^{\frac{1}{2}}(c / d)^{\frac{1}{2}},-\epsilon^{-1} q^{\frac{1}{2}}(d / c)^{\frac{1}{2}}, \\
\left.-\epsilon q^{\frac{1}{2}} b(c / d)^{\frac{1}{2}} ; q, q^{k}\right)=P_{\lambda}^{B}\left(z ; a, b, c, d ; q, q^{k}\right) .
\end{array}
$$

(b) Fix $q \in(0,1)$ and $k \in \mathbf{N}$. Suppose that $(a, b) \in V_{L}^{q}$, then

$$
\lim _{\epsilon \downarrow 0}\left(\frac{\epsilon}{q^{\frac{1}{2}}}\right)^{|\lambda|} P_{\lambda}^{A W}\left(\frac{q^{\frac{1}{2}} z}{\epsilon} ; \epsilon q^{\frac{1}{2}} b, \epsilon^{-1} q^{\frac{1}{2}},-q^{\frac{1}{2}},-q^{\frac{1}{2}} a ; q, q^{k}\right)=P_{\lambda}^{L}\left(z ; a, b ; q, q^{k}\right) .
$$

Pr o of. The essence of the proof can be found in [SK]. The theorem was proved in [SK] for a dense subset of the parameter domain, making use of a definition of Askey-Wilson polynomials for general parameter values by rational extension in the parameters. Let us first give the idea of the proof. It follows from theorem 2.4 that

$$
P_{\lambda}^{A W}=\left(\prod_{\mu<\lambda} \frac{D_{A W}-E_{\mu}^{A W}}{E_{\lambda}^{A W}-E_{\mu}^{A W}}\right) m_{\lambda}
$$

for parameter values such that $E_{\mu}^{A W} \neq E_{\lambda}^{A W}$ for all $\mu<\lambda$. Furthermore we have

$$
P_{\lambda}^{L}=\left(\prod_{\mu<\lambda} \frac{D_{L}-E_{\mu}^{B, L}}{E_{\lambda}^{B, L}-E_{\mu}^{B, L}}\right) \tilde{m}_{\lambda}
$$

for parameter values such that $E_{\mu}^{B, L} \neq E_{\lambda}^{B, L}$ for all $\mu<\lambda$ and similarly for the big q-Jacobi case. For these parameter values, the limit transition can be proved by taking limits of $D_{A W}$ and $E_{\lambda}^{A W}$. This gives the result for a dense subset of the parameter domain. Since we know now the orthogonality measure for the larger parameter domain $V_{A W}$, we can prove the limit transitions for all parameter values by a type of continuity argument (section 6 in $[\mathrm{SK}]$ ). We have to slightly modify the arguments of (section 6 , $[\mathrm{SK}]$ ) for the proof of this theorem, because we work with discrete parameter $t$. We give the proof of (b), the proof of (a) is similar.

(b) For arbitrary $(a, b) \in \mathbf{R}^{2}$, let $V_{L}(a, b)$ be the open subset of $(0,1)$ defined by $V_{L}(a, b):=\left\{q \in(0,1) /(a, b) \in V_{L}^{q}\right\}$. Fix $(a, b) \in \mathbf{R}^{2}$ with $V_{L}(a, b) \neq \emptyset$. Fix $k \in \mathbf{N}$. There exists an $\epsilon_{L}>0$, independent of $q$, such that

$$
\left(a_{L}(\epsilon), b_{L}(\epsilon), c_{L}(\epsilon), d_{L}(\epsilon)\right):=\left(\epsilon q^{\frac{1}{2}} b, \epsilon^{-1} q^{\frac{1}{2}},-q^{\frac{1}{2}},-q^{\frac{1}{2}} a\right) \in V_{A W}
$$

for all $\epsilon \in\left(0, \epsilon_{L}\right)$ and all $q \in V_{L}(a, b)$. Hence the left hand side of formula (3.7) makes sense. Denote $m_{\nu, \epsilon}(z):=\left(\epsilon / q^{\frac{1}{2}}\right)^{|\nu|} m_{\nu}\left(q^{\frac{1}{2}} z / \epsilon\right)$, then we have that

$$
\begin{aligned}
P_{\lambda}^{A W, L}(z ; \epsilon) & :=\left(\frac{\epsilon}{q^{\frac{1}{2}}}\right)^{|\lambda|} P_{\lambda}^{A W}\left(\frac{q^{\frac{1}{2}} z}{\epsilon} ; a_{L}(\epsilon), b_{L}(\epsilon), c_{L}(\epsilon), d_{L}(\epsilon) ; q, q^{k}\right) \\
& =m_{\lambda, \epsilon}(z)+\sum_{\nu<\lambda} d_{\lambda, \nu}^{A W, L}\left(a, b ; q, q^{k} ; \epsilon\right) m_{\nu, \epsilon}(z)
\end{aligned}
$$


for certain constants $d_{\lambda, \nu}^{A W, L}$ if $\epsilon \in\left(0, \epsilon_{L}\right)$ and $q \in V_{L}(a, b)$. By definition, there exist constants $d_{\lambda, \nu}^{L}$ such that

$$
P_{\lambda}^{L}\left(z ; a, b ; q, q^{k}\right)=\tilde{m}_{\lambda}(z)+\sum_{\nu<\lambda} d_{\lambda, \nu}^{L}\left(a, b ; q, q^{k}\right) \tilde{m}_{\nu}(z)
$$

for $q \in V_{L}(a, b)$. It will be sufficient to prove that

$$
\lim _{\epsilon \downarrow 0} d_{\lambda, \nu}^{A W, L}\left(a, b ; q, q^{k} ; \epsilon\right)=d_{\lambda, \nu}^{L}\left(a, b ; q, q^{k}\right)
$$

for all $\nu, \lambda \in P^{+}$with $\nu<\lambda$ and for all $q \in V_{L}(a, b)$, because

$$
\lim _{\epsilon \rightarrow 0} m_{\nu, \epsilon}=\tilde{m}_{\nu} \quad \forall \nu \in P^{+} .
$$

Fix $\nu, \lambda \in P^{+}$with $\nu<\lambda$. The proof of (3.13) consists of five steps:

(i) Define

$$
\tilde{V}_{L, \lambda}(a, b):=\left\{q \in V_{L}(a, b) / E_{\lambda}^{B, L}\left(a, b ; q, q^{k}\right) \neq E_{\mu}^{B, L}\left(a, b ; q, q^{k}\right) \quad \forall \mu<\lambda\right\},
$$

then $\tilde{V}_{L, \lambda}(a, b) \subset V_{L}(a, b)$ is dense.

(ii) $d_{\lambda, \nu}^{A W, L}\left(a, b ; q, q^{k} ; \epsilon\right)$ depends polynomially on $\epsilon$ for $q \in \tilde{V}_{L, \lambda}(a, b)$.

(iii) $\lim _{\epsilon \downarrow 0} d_{\lambda, \nu}^{A W, L}\left(a, b ; q, q^{k} ; \epsilon\right)=d_{\lambda, \nu}^{L}\left(a, b ; q, q^{k}\right)$ for all $q \in \tilde{V}_{L, \lambda}(a, b)$.

(iv) The map $q \mapsto d_{\lambda, \nu}^{A W, L}\left(a, b ; q, q^{k} ; \epsilon\right): V_{L}(a, b) \rightarrow \mathbf{C}$ is continuous for all $\epsilon \in\left(0, \epsilon_{L}\right)$.

(v) The map $q \mapsto d_{\lambda, \nu}^{L}\left(a, b ; q, q^{k}\right): V_{L}(a, b) \rightarrow \mathbf{C}$ is continuous.

It follows easily from (i), (ii) and (iv) that the limit $\lim _{\epsilon \downarrow 0} d_{\lambda, \mu}^{A W, L}\left(a, b ; q, q^{k} ; \epsilon\right)$ exists for all $q \in V_{L}(a, b)$ and that the limit depends continuously on $q \in V_{L}(a, b)$ (cf. [SK], lemma 6.1). (iii) and (v) imply then that the limit will be $d_{\lambda, \nu}^{L}\left(a, b ; q, q^{k}\right)$ for all $q \in V_{L}(a, b)$, hence (b) of the theorem follows.

We proceed with proving the five steps. Fix $\nu, \lambda \in P^{+}$with $\nu<\lambda$.

(i) It is sufficient to prove that for arbitrary $a, b \in \mathbf{C}$ and arbitrary $\lambda, \mu \in P^{+}$, $E_{\lambda}^{B, L}\left(a, b ; q, q^{k}\right)=E_{\mu}^{B, L}\left(a, b ; q, q^{k}\right)$ as Laurent polynomials in $q$ if and only if $\lambda=\mu$. If $E_{\lambda}^{B, L}\left(a, b ; q, q^{k}\right)=E_{\mu}^{B, L}\left(a, b ; q, q^{k}\right)$ as Laurent polynomials in $q$, then

$$
(a b) \sum_{i=1}^{n}\left(q^{\lambda_{i}+k(n-i)+1}-q^{\mu_{i}+k(n-i)+1}\right)=\sum_{i=1}^{n}\left(q^{-\mu_{i}-k(n-i)}-q^{-\lambda_{i}-k(n-i)}\right)
$$

as Laurent polynomials in $q$. This implies that

$$
\sum_{i=1}^{n} q^{-\mu_{i}-k(n-i)}=\sum_{i=1}^{n} q^{-\lambda_{i}-k(n-i)}
$$

as Laurent polynomials in $q$. Since $\mu, \lambda \in P^{+}$, we get $\mu_{i}+k(n-i)=\lambda_{i}+k(n-i)$ for all $i$. Hence $\lambda=\mu$.

(ii) Denote $D_{A W, L}^{\epsilon}$ for the q-difference operator $D_{A W, q, q^{k}}^{\epsilon q^{\frac{1}{2}} b, \epsilon^{-1} q^{\frac{1}{2}},-q^{\frac{1}{2}},-q^{\frac{1}{2}} a}$. We have

$$
E_{\lambda}^{A W}\left(a_{L}(\epsilon), b_{L}(\epsilon), c_{L}(\epsilon), d_{L}(\epsilon) ; q, q^{k}\right)=E_{\lambda}^{B, L}\left(a, b ; q, q^{k}\right)
$$

independent of $\epsilon$ for all $\lambda \in P^{+}$, so we can write $P_{\lambda}^{A W, L}(z ; \epsilon)$ for $q \in \tilde{V}_{L, \lambda}(a, b)$ as

$$
P_{\lambda}^{A W, L}(. ; \epsilon)=\left(\prod_{\mu<\lambda} \frac{D_{A W, L}^{\epsilon}-E_{\mu}^{B, L}}{E_{\lambda}^{B, L}-E_{\mu}^{B, L}}\right) m_{\lambda, \epsilon} .
$$


Hence it is sufficient to prove that the coefficients $\tilde{c}_{\lambda, \nu}^{A W, L}(\epsilon)(\nu \leq \lambda)$ in the expansion

$$
D_{A W, L}^{\epsilon} m_{\lambda, \epsilon}=\sum_{\nu \leq \lambda} \tilde{c}_{\lambda, \nu}^{A W, L}(\epsilon) m_{\nu, \epsilon}
$$

depend polynomially on $\epsilon$. The coefficients can be written as

$$
\tilde{c}_{\lambda, \nu}^{A W, L}(\epsilon)=\left(\frac{\epsilon}{q^{\frac{1}{2}}}\right)^{|\lambda|-|\nu|} c_{\lambda, \nu}^{A W}\left(\epsilon q^{\frac{1}{2}} b, \epsilon^{-1} q^{\frac{1}{2}},-q^{\frac{1}{2}},-q^{\frac{1}{2}} a ; q, q^{k}\right)
$$

where $c_{\lambda, \nu}^{A W}(\nu \leq \lambda)$ are the coefficients in the expansion

$$
D_{A W} m_{\lambda}=\sum_{\nu \leq \lambda} c_{\lambda, \nu}^{A W} m_{\nu}
$$

In $[\mathrm{SK}]$, prop. $5.3(\mathbf{2})$, it was proven that the coefficients $\tilde{c}_{\lambda, \nu}^{A W, L}(\epsilon)(\nu \leq \lambda)$ depend polynomially on $\epsilon$.

(iii) Follows from $(3.16),(3.14)$ and $(3.9)$, since the constant terms $c_{\lambda, \nu}^{L}(\nu \leq \lambda)$ of the polynomials $\tilde{c}_{\lambda, \nu}^{A W, L}(\epsilon)(\nu \leq \lambda)$ are exactly the expansion coefficients of $D_{L, q, q^{k}}^{a, b} \tilde{m}_{\lambda}$ with respect to the basis $\left\{\tilde{m}_{\mu} / \mu \in P^{+}\right\}$:

$$
D_{L, q, q^{k}}^{a, b} \tilde{m}_{\lambda}=\sum_{\nu \leq \lambda} c_{\lambda, \nu}^{L} \tilde{m}_{\nu}
$$

(cf. [SK], theorem 5.1(2) and prop. $5.3(2)$ ).

(iv) The map

$$
q \mapsto\left\langle m_{\rho}, m_{\sigma}\right\rangle_{A W, q, q^{k}}^{\epsilon q^{\frac{1}{2}} b, \epsilon^{-1} q^{\frac{1}{2}},-q^{\frac{1}{2}},-q^{\frac{1}{2}} a}: V_{L}(a, b) \rightarrow \mathbf{C}
$$

is continuous for all $\rho, \sigma \in P^{+}$and all $\epsilon \in\left(0, \epsilon_{L}\right)$. Hence

$$
q \mapsto d_{\lambda, \nu}^{A W, L}\left(a, b ; q, q^{k} ; \epsilon\right): V_{L}(a, b) \rightarrow \mathbf{C}
$$

is continuous for all $\epsilon \in\left(0, \epsilon_{L}\right)$ (cf. [SK], prop. 2.3(a)).

(v) The continuity of the map

$$
q \mapsto d_{\lambda, \nu}^{L}\left(a, b ; q, q^{k}\right): V_{L}(a, b) \rightarrow \mathbf{C}
$$

follows from the continuity of the maps

$$
q \mapsto\left\langle\tilde{m}_{\rho}, \tilde{m}_{\sigma}\right\rangle_{L, q, q^{k}}^{a, b}: V_{L}(a, b) \rightarrow \mathbf{C} \quad\left(\rho, \sigma \in P^{+}\right) .
$$

This completes the proof of the theorem.

The behaviour of the support of the orthogonality measures under these limit transitions is similar to the behaviour in the one variable case (cf. [K2]). Indeed, for the limit transition to the big q-Jacobi polynomials, fix $q \in(0,1)$ and $(a, b, c, d) \in V_{B}^{q}$. Fix $k \in \mathbf{N}$. Then there exists an $\epsilon_{B}>0$ such that

$$
\begin{aligned}
\left(a_{B}(\epsilon), b_{B}(\epsilon), c_{B}(\epsilon), d_{B}(\epsilon)\right):=\left(\epsilon q^{\frac{1}{2}} a(d / c)^{\frac{1}{2}},\right. & \epsilon^{-1} q^{\frac{1}{2}}(c / d)^{\frac{1}{2}} \\
& \left.-\epsilon^{-1} q^{\frac{1}{2}}(d / c)^{\frac{1}{2}},-\epsilon q^{\frac{1}{2}} b(c / d)^{\frac{1}{2}}\right) \in V_{A W}
\end{aligned}
$$

for $\epsilon \in\left(0, \epsilon_{B}\right)$. Denote

$$
P_{\lambda}^{A W, B}(z ; \epsilon):=\left(\frac{\epsilon(c d)^{\frac{1}{2}}}{q^{\frac{1}{2}}}\right)^{|\lambda|} P_{\lambda}^{A W}\left(\frac{q^{\frac{1}{2}} z}{\epsilon(c d)^{\frac{1}{2}}} ; a_{B}(\epsilon), b_{B}(\epsilon), c_{B}(\epsilon), d_{B}(\epsilon) ; q, q^{k}\right)
$$


then the support of the orthogonality measure of $\left\{P_{\lambda}^{A W, B}(z ; \epsilon) / \lambda \in P^{+}\right\}$for $\epsilon \in\left(0, \epsilon_{B}\right)$ small enough, is essentially given by (cf. lemma 2.2)

$$
\begin{array}{r}
\bigcup_{m=0}^{n}\left\{z \in \mathbf{C}^{n} / z_{l} \in\left\{-d q^{r_{1}}\right\}_{r_{1}=0}^{N_{1, \epsilon}} \cup\left\{c q^{r_{2}}\right\}_{r_{2}=0}^{N_{2, \epsilon}}(l=1, \ldots, m)\right. \text { such that } \\
z_{i} \neq q^{r} z_{j} \text { for } 1 \leq i \neq j \leq m \text { and for } r=0, \ldots, k-1, \\
\text { and } \left.\left|z_{l}\right|=\epsilon(c d / q)^{\frac{1}{2}} \quad(l=m+1, \ldots, n)\right\}
\end{array}
$$

with $N_{1, \epsilon}, N_{2, \epsilon} \in \mathbf{N}_{0}$ the largest integers such that

$$
\left|\epsilon^{-1} q^{\frac{1}{2}}(d / c)^{\frac{1}{2}} q^{N_{1, \epsilon}}\right|>1 \text {, resp. }\left|\epsilon^{-1} q^{\frac{1}{2}}(c / d)^{\frac{1}{2}} q^{N_{2, \epsilon}}\right|>1 .
$$

We will give now a formal calculation in order to see which weights tend (formally) to non zero weights under the limit transition. We will rescale the weights (rescaling factor depends on $\epsilon$ ) for the Askey-Wilson polynomials involved in the limit in such a way, that the formal limits $\epsilon \downarrow 0$ of the rescaled weights exist and are not all identically zero. The formal limit we will use, is the following. Let $a_{1}, \ldots, a_{r}, b_{1}, \ldots, b_{s} \in \mathbf{C} \backslash\{0\}$, then we assume that

$$
\lim _{x \rightarrow \infty} \frac{\left(a_{1} x, \ldots, a_{r} x ; q\right)_{\infty}}{\left(b_{1} x, \ldots, b_{s} x ; q\right)_{\infty}}=0
$$

if $r<s$ or if $r=s$ and $\left|a_{1} \ldots a_{r}\right|<\left|b_{1} \ldots b_{r}\right|$. In order words, writing the quotient of infinite products in (3.18) as

$$
\prod_{j=0}^{\infty} \frac{\left(1-a_{1} q^{j} x\right) \ldots\left(1-a_{r} q^{j} x\right)}{\left(1-b_{1} q^{j} x\right) \ldots\left(1-b_{s} q^{j} x\right)}
$$

we assume that we may take the limit termwise within the infinite product. Denote

$$
z^{(m)}(\epsilon):=\left(z_{1}, \ldots, z_{m}, \epsilon(c d / q)^{\frac{1}{2}} c_{m+1}, \ldots, \epsilon(c d / q)^{\frac{1}{2}} c_{n}\right),
$$

where $m \in\{0, \ldots, n\},\left|c_{m+1}\right|=\ldots=\left|c_{n}\right|=1$ and $z_{l} \in\left\{-d q^{r}, c q^{s}\right\}_{r, s \in \mathbf{N}_{0}}(l=1, \ldots, m)$ such that $z_{i} \neq q^{r} z_{j}$ for $1 \leq i \neq j \leq m$ and for $r=0, \ldots, k-1$. $z^{(m)}(\epsilon)$ is in the support of the orthogonality measure for the polynomials $\left\{P_{\lambda}^{A W, B}(z ; \epsilon) / \lambda \in P^{+}\right\}$if $\epsilon$ is sufficiently small. The corresponding (rescaled) weight is given by

$$
\begin{aligned}
& \Delta_{m}^{B}\left(z^{(m)}(\epsilon)\right) \\
& \quad:=\left(-\epsilon^{-2} q ; q\right)_{\infty}^{n} \epsilon^{k n(n-1)} \Delta_{A W, m}\left(\frac{q^{\frac{1}{2}} z^{(m)}(\epsilon)}{\epsilon(c d)^{\frac{1}{2}}} ; a_{B}(\epsilon), b_{B}(\epsilon), c_{B}(\epsilon), d_{B}(\epsilon) ; q, q^{k}\right) .
\end{aligned}
$$

A straightforward calculation, using the formal limit (3.18), gives that

$$
\begin{gathered}
\lim _{\epsilon \downarrow 0} \Delta_{m}^{B}\left(z^{(m)}(\epsilon)\right)=0 \text { if } 0 \leq m<n, \\
\lim _{\epsilon \downarrow 0} \Delta_{n}^{B}\left(z^{(n)}(\epsilon)\right)=K_{B} \Delta_{B}(z) \prod_{j=1}^{n}\left|z_{j}\right|,
\end{gathered}
$$

with $K_{B}$ a positive constant given by

$$
K_{B}:=(q ; q)_{\infty}^{-2 n}(-d / c,-c / d ; q)_{\infty}^{-n}\left(\frac{c+d}{c d}\right)^{n} q^{k^{2}\left(\begin{array}{c}
n \\
2
\end{array}\right)}(c d)^{-k\left(\begin{array}{c}
n \\
2
\end{array}\right)} .
$$


Since $N_{1, \epsilon}, N_{2, \epsilon} \rightarrow \infty$ if $\epsilon \downarrow 0$, we see that formally in the limit $\epsilon \downarrow 0$, the part of the measure with (partially) continuous support disappears while the completely discrete part of the orthogonality blows up to the set

$$
\begin{aligned}
& \left\{z \in \mathbf{R}^{n} / z_{i} \in\left\{-d q^{r}, c q^{s}\right\}_{r, s \in \mathbf{N}_{0}} \quad(i=1, \ldots, n)\right. \text { such that } \\
& \left.z_{i} \neq q^{l} z_{j} \text { for } 1 \leq i \neq j \leq n \text { and for } l=0, \ldots, k-1\right\} .
\end{aligned}
$$

We recognize the set (3.19) as the support of the orthogonality measure for the big q-Jacobi polynomials $\left\{P_{\lambda}^{B}\left(z ; a, b, c, d ; q, q^{k}\right) / \lambda \in P^{+}\right\}$(cf. lemma 3.1), and the corresponding formal weights are (up to a positive constant) equal to the weights for the big q-Jacobi polynomials.

A similar interpretation can be given for the the limit from Askey-Wilson polynomials to little q-Jacobi polynomials. Fix $q \in(0,1),(a, b) \in V_{L}^{q}$ and $k \in \mathbf{N}$. The support of the orthogonality measure for the polynomials $\left\{P_{\lambda}^{A W, L}\left(z ; a, b ; q, q^{k} ; \epsilon\right) / \lambda \in P^{+}\right\}$(cf. (3.11)) for $\epsilon$ sufficient small is given by

$$
\begin{aligned}
& \bigcup_{m=0}^{n}\left\{z \in \mathbf{C}^{n} / z_{l} \in V_{a}(\epsilon)(l=1, \ldots, m) \text { such that } z_{i} \neq q^{r} z_{j} \text { for } 1 \leq i \neq j \leq m\right. \\
& \text { and for } \left.r=0, \ldots, k-1, \text { and }\left|z_{l}\right|=\epsilon / q^{\frac{1}{2}} \quad(l=m+1, \ldots, n)\right\}
\end{aligned}
$$

where $V_{a}(\epsilon)=\left\{q^{r}\right\}_{r=0}^{N_{\epsilon}} \cup\{-\epsilon a\}$ if $\left|a q^{\frac{1}{2}}\right|>1$ resp. $V_{a}(\epsilon)=\left\{q^{r}\right\}_{r=0}^{N_{\epsilon}}$ if $\left|a q^{\frac{1}{2}}\right| \leq 1$ and with $N_{\epsilon} \in \mathbf{N}_{0}$ largest such that $\left|\epsilon^{-1} q^{\frac{1}{2}} q^{N_{\epsilon}}\right|>1$. Denote

$$
z^{(m)}(\epsilon):=\left(z_{1}, \ldots, z_{m}, c_{m+1} \epsilon / q^{\frac{1}{2}}, \ldots, c_{n} \epsilon / q^{\frac{1}{2}}\right)
$$

where $m \in\{0, \ldots, n\},\left|c_{m+1}\right|=\ldots=\left|c_{n}\right|=1$ and $z_{l} \in\left\{q^{r}\right\}_{r \in \mathbf{N}_{0}} \cup\{-\epsilon a\}$ if $\left|a q^{\frac{1}{2}}\right|>1$ resp. $z_{l} \in\left\{q^{l}\right\}_{l \in \mathbf{N}_{0}}$ if $\left|a q^{\frac{1}{2}}\right| \leq 1(l=1, \ldots, m)$, such that $z_{i} \neq q^{r} z_{j}$ for $1 \leq i \neq j \leq m$ and for $r=0, \ldots, k-1$. $z^{(m)}(\epsilon)$ is in the support of the orthogonality measure for the polynomials $\left\{P_{\lambda}^{A W, L}(z ; \epsilon) / \lambda \in P^{+}\right\}$if $\epsilon$ is sufficiently small. The corresponding (rescaled) weight is then given by

$$
\begin{array}{r}
\Delta_{m}^{L}\left(z^{(m)}(\epsilon)\right):=\left(-\epsilon^{-1} q,-\epsilon^{-1} q a ; q\right)_{\infty}^{n} \epsilon^{k n(n-1)} \Delta_{A W, m}\left(\frac{q^{\frac{1}{2}} z^{(m)}(\epsilon)}{\epsilon} ; a_{L}(\epsilon), b_{L}(\epsilon),\right. \\
\left.\quad c_{L}(\epsilon), d_{L}(\epsilon) ; q, q^{k}\right),
\end{array}
$$

with $\left(a_{L}(\epsilon), b_{L}(\epsilon), c_{L}(\epsilon), d_{L}(\epsilon)\right)$ given by (3.10). A straightforward calculation, using the formal limit (3.18), gives then that

$$
\begin{gathered}
\lim _{\epsilon \downarrow 0} \Delta_{m}^{L}\left(z^{(m)}(\epsilon)\right)=0 \quad \text { if } 0 \leq m<n, \\
\lim _{\epsilon \downarrow 0} \Delta_{n}^{L}\left(z^{(n)}(\epsilon)\right)=0 \quad \text { if } z_{i}=-\epsilon a \text { for certain } i \in\{1, \ldots, n\}, \\
\lim _{\epsilon \downarrow 0} \Delta_{n}^{L}\left(z^{(n)}(\epsilon)\right)=K_{L} \Delta_{L}(z) \prod_{j=1}^{n} z_{j} \quad \text { if } z_{i} \neq-\epsilon a \forall i \in\{1, \ldots, n\}
\end{gathered}
$$


with $K_{L}$ a positive constant given by

$$
K_{L}:=(q ; q)_{\infty}^{-2 n} q^{k^{2}\left(\begin{array}{c}
n \\
2
\end{array}\right)}
$$

Since $N_{\epsilon} \rightarrow \infty$ if $\epsilon \downarrow 0$, we see that formally in the limit $\epsilon \downarrow 0$ the part of the orthogonality measure with (partially) continuous support disappears while the completely discrete part of the orthogonality blows up to the set

$$
\begin{aligned}
\left\{z \in \mathbf{R}^{n} / z_{i} \in\left\{q^{s}\right\}_{s \in \mathbf{N}_{0}}\right. & (i=1, \ldots, n) \text { such that } z_{i} \neq q^{l} z_{j} \\
& \text { for } 1 \leq i \neq j \leq n \text { and for } l=0, \ldots, k-1\} .
\end{aligned}
$$

The set given by (3.20) is exactly the support of the orthogonality measure for the little q-Jacobi polynomials $\left\{P_{\lambda}^{B}\left(z ; a, b, c, d ; q, q^{k}\right) / \lambda \in P^{+}\right\}$(cf. lemma 3.3), and the corresponding formal weights are (up to a positive constant) equal to the weights for the little q-Jacobi polynomials.

\section{References}

[AA] G.E. Andrews, R. Askey, Classical orthogonal polynomials, in: Polynômes Orthogonaux et Applications, C. Brezinski, A. Draux, A.P. Magnus, P. Maroni and A. Ronveaux, eds., Lecture Notes in Math. 1171, Springer, New York, 1985, 36-62.

[AW] R. Askey, J. Wilson, Some basic hypergeometric orthogonal polynomials that generalize Jacobi polynomials, Mem. Amer. Math. Soc. 54 (1985), no. 319.

[K1] T.H. Koornwinder, Askey-Wilson polynomials for root systems of type BC, Contemp. Math. 138 (1992), 189-204.

[K2] T.H. Koornwinder, Askey-Wilson polynomials as zonal spherical functions on the SU(2) quantum group, SIAM J. Math. Anal. 24 (1993), pp. 795-813.

[M] I.G. Macdonald, Orthogonal polynomials associated with root systems, preprint 1988.

[S] J.V.Stokman, Multivariable big and little q-Jacobi polynomials, Mathematical preprint series, Univ. of Amsterdam (1995), report 95-16, to appear in SIAM J. Math. Anal.

[S1] J.V. Stokman, Askey-Wilson polynomials with partly discrete ortogonality measure, Mathematical preprint series, Univ. of Amsterdam (1996), report 96-06, to appear in the Ramanujan Journal.

[SK] J.V. Stokman, T.H. Koornwinder, Limit transitions for BC type multivariable orthogonal polynomials, Mathematical preprint series, Univ. of Amsterdam (1995), report 95-19. To appear in Canad. J. Math. 